\title{
PRODUTOS CIENTÍFICO E TECNOLÓGICO DO GRUPO DE PESQUISA EM MATERIAIS COMPÓSITOS DA FACULDADE DE ENGENHARIA MECÂNICA DA UFPA: IC's, TCC's, ARTIGOS E PATENTES
}

\author{
DOI: 10.37702/2175-957X.COBENGE.2021.3496
}

Léo César de Oliveira Pereira - leocesaroliveira@hotmail.com

Universidade Federal do Pará

Conjunto Guajará I Tv WE 63A 1421

67143-390 - Ananindeua - PA

Roberto Tetsuo Fujiyama - fujiyama@ufpa.br

Universidade Federal do Pará

AV. TAVARES BASTOS 438/203

66613-005 - BELEM - PA

Resumo: A Universidade Federal do Pará (UFPA) tem como missão formar pessoas com um caráter sustentável e gerar produção científica e tecnológica para destacar a importância deste tema é fundamental. O Grupo de Pesquisa em Materiais Compósitos (GPMAC) da Faculdade de Engenharia Mecânica da UFPA utiliza destas premissas em seus trabalhos com compósitos reforçados com fibras vegetais e resíduos industriais para formar seus alunos em todos os graus de docência, tentando estabelecer este elo desde a iniciação científica (IC) até o doutorado e este trabalho visa identificar, através de um estudo de caso quantitativo, a contribuição da produção científica e tecnológica do GPMAC referente à IC's, Trabalhos de Conclusão de Curso, Dissertações e Teses, bem como a quantidade de publicação científica do grupo em periódicos, anais de eventos, capítulos de livro e em especial referente às patentes. Observou-se que a Iniciação Científica é uma atividade precursora para as atividades de Trabalho Final de Curso, tanto para a graduação como para a pós-graduação. Os Trabalhos Finais de Curso de graduação foram em maior quantidade seguida de dissertações de mestrado. No que se refere a produção bibliográfica, foi observado que a maior quantidade de artigos publicados foi em congressos nacionais e internacionais, seguido de publicações em periódicos. $E$ a produção de inovação tecnológica através de patentes foi 3,14\% do total de publicações. 


\section{COBENCE \\ $2021 \begin{aligned} & \text { eIV Simpósio Internacional } \\ & \text { de Educação em Engenharia } \\ & \text { da ABENGE }\end{aligned}$ 28 a 30 de SETEMBRO \\ Formação em Engenharia: Tecnologia, Inovaçáo e Sustentabilidade"}

Palavras-chave: Sustentabilidade, Produção científica e tecnológica, Grupo de pesquisa. 


\section{INTRODUÇÃO}

A Universidade Federal do Pará, de forma geral, se propõe em seu Projeto de Desenvolvimento Institucional (PDI), (UFPA, 2016), no que diz respeito à sua filosofia de trabalho, à missão, às diretrizes pedagógicas que orientam suas ações, à sua estrutura organizacional e às atividades acadêmicas que desenvolve e/ou que pretende desenvolver para cumprir sua missão de produzir, socializar e transformar o conhecimento na Amazônia para a formação de cidadãos capazes de promover a construção de uma sociedade sustentável. Com esta premissa se objetiva desenvolver no discente um caráter sustentável em suas atividades, projetos e ações durante o curso para a permanência desta postura após seu egresso.

No que tange a educação superior e a ênfase na pesquisa acadêmica, em especial se tratando das Iniciações científicas e atividades de extensão, o Plano Nacional de Graduação (PNG) de 2001 (BRASIL, 2001) aponta para a importância da pesquisa na graduação por constituir um espaço em que o aluno atua como sujeito de sua aprendizagem, por meio, principalmente, da integração entre a graduação e a pósgraduação. Segundo Brasil (2001, p. 12), todo esse processo visa atingir a indissociabilidade entre pesquisa, ensino e extensão, como condição para o exercício profissional criativo.

Estas diretrizes já se faziam presentes na Recomendação Normativa (CNPq. RN005/1993), que alerta que as atividades da IC são importantes devido a várias razões, como por exemplo: a) despertar vocação científica e incentivar talentos potenciais entre estudantes de graduação, mediante suas participações em projetos de pesquisa, introduzindo o jovem universitário no domínio do método científico; b) proporcionar ao bolsista, orientado por pesquisador qualificado, a aprendizagem de técnicas e métodos científicos, bem como estimular o desenvolvimento do pensar cientificamente e da criatividade, decorrentes das condições criadas pelo confronto direto com os problemas de pesquisa; c) possibilitar a diminuição do tempo de permanência do bolsista na pósgraduação; d) despertar no bolsista uma nova mentalidade em relação à pesquisa e prepará-lo para a pós-graduação.

A Faculdade de Engenharia Mecânica está localizada no Instituto de Tecnologia, onde também se encontram outras Faculdades de Engenharia (Elétrica, Química, Civil. Naval, Alimentos, Computação e Telecomunicações, Sanitária e Ambiental, Ferroviária e Logística e de Conservação e Restauro) segundo (ITEC, 2021).

O Projeto Pedagógico do Curso (PPC) de Engenharia Mecânica da FEM permite o acesso dos alunos aos seguintes laboratórios conforme FEM, (FEM, 2021):

- Laboratórios da Área de Materiais e Processos de Fabricação: Caracterização de Materiais, Processamento de Materiais, Ensaios Mecânicos, Máquinas Operatrizes, Materiais Compósitos, Metalografia e Tratamento Térmico, Metrologia, Processamento e Caracterização de Soldas e Solidificação de Metais,

- Laboratório da Área de Sistemas Mecânicos: Acústica, Cinemática e Dinâmica de Máquinas, Vibrações Mecânicas, Laboratório de Mecânica Computacional, Laboratório de Comportamento Mecânico dos Materiais,

- Laboratórios da Área de Térmica e Fluidos - Combustíveis e Combustão, Climatização e Conforto Térmico, Energias Renováveis, Mecânica dos Fluidos, Motores de Combustão Interna, Refrigeração, Transferência de Calor e Massa, Turbomáquinas e Vapor;

\section{- Laboratórios da Área de Gestão e Produção - Lubrificação e Manutenção.}


O Grupo de Pesquisa em Materiais Compósitos (GPMAC) da Faculdade de Engenharia Mecânica (FEM) da Universidade Federal do Pará é um dos 9 grupos de estudos vinculados ao Laboratório de Engenharia Mecânica (LABEM). No LABEM são desenvolvidas as atividades de pesquisa e desenvolvimento tecnológico do curso de Engenharia Mecânica voltados às áreas de térmicas e fluidos, materiais e processos de fabricação, sistemas mecânicos e gestão e manutenção.

O Laboratório do Grupo de Pesquisa em Materiais Compósitos: Tem infraestrutura para apoiar discentes da graduação e pós-graduação. Os principais equipamentos nele instalados são balança elétrica de precisão, máquina de soldagem elétrica, moto esmeril, furadeira manual, poli corte manual, soprador térmico, moldes de madeira, moldes de silicone, compressor de 1HP para pintura de precisão, compressor de 2 HP para uso geral, máquina de vácuo para processo de infusão e processos similares, prensa hidráulica, furadeira de bancada, equipamentos específicos para corte e preparação de amostras de plásticos e materiais diversos de acordo com normas da ASTM, forno elétrico para secagem de matéria orgânica e cura de a mostras de pequenas dimensões, forno elétrico para secagem de matéria orgânica e cura de amostras de grandes dimensões e cortador com resistência elétrica para corte de isopor com alta precisão.

O GPMAC faz parte da área de estudos de materiais e processos de fabricação e utiliza de resíduos da agroindústria, indústrias de minério e madeireiras, bem como de fibras vegetais tradicionais da região amazônica com a finalidade de reaproveitar e/ou agregar valor às fibras ou resíduos, gerando um ciclo que envolve o desenvolvimento sustentável de novos materiais com propriedades físicas e mecânicas que atendam às necessidades de fabricação de diversos produtos. Fazendo do compósito polimérico reforçado com as fibras e resíduos vegetais o seu ramo de pesquisa principal. O GPMAC iniciou as suas atividades com compósitos em 1999.

Apesar da importância da IC, para a grande maioria dos discentes, o Trabalho de Conclusão de Curso (TCC) é a primeira e única produção científica construída ao longo dos anos na experiência da Graduação. Trata-se de uma construção que segue um rigor epistemológico, estrutural e metodológico, pautados por normas de trabalho acadêmico, adotadas pelas instituições de ensino superior. Entretanto, é importante ressaltar que, tanto a instituição formadora quanto os alunos precisam estar imbuídos do mesmo espírito científico, crítico e participativo, tão essenciais para a elaboração de um TCC, ou seja, que mecanismos de reflexão, orientação, acompanhamento e de avaliação façam parte dessa rotina acadêmica (OLIVEIRA, 2003).

$\mathrm{Na}$ UFPA a pós-graduação conta com o Programa de Pós Graduação em Engenharia Mecânica (PPGEM) que assume as diretrizes do Mestrado em engenharia mecânica e o Programa de Pós-Graduação em Engenharia de Recursos Naturais da Amazônia (PRODERNA) que é responsável pelo Doutorado nas engenharias no que tange a utilização de recursos naturais. A pós-graduação tem sua importância no papel acadêmico e o Sistema Nacional da Pós-Graduação (SNPG) estabelece duas metas centrais: formar competentes professores com qualidade e atender às necessidades do ensino, além de formar excelentes pesquisadores no campo das ciências básicas e das ciências aplicadas. Espera-se deste profissional que domínios epistemológicos tenham sido incorporados a ele e ajudem no desenvolvimento de projetos de pesquisa, assim como aspectos pedagógicos da aprendizagem e formação ligados ao se tornar pesquisador e aprender a orientar possam ter sidos sistematizados (FREITAS, SOUZA, 2018)

Como parte complementar das atividades dos grupos de estudos o resultado dos trabalhos acadêmicos é publicado em veículos de comunicação. Neste contexto, a produção científica é de suma importância pois a avaliação da qualidade acadêmica de um cientista é possibilitada por meio de sua produção científica. A informação científica é tida 
como insumo básico para o desenvolvimento científico de cada país e para o progresso mundial (DROESCHER; SILVA, 2014). As publicações científicas objetivam divulgar a pesquisa para a comunidade, de forma que permita que outros possam utilizá-la e avaliala sob outras visões, ou seja, é por meio dela que a sociedade toma conhecimento dos resultados de um trabalho de pesquisa e o que ele representa para a coletividade (BROFMAN, 2018). Estas produções podem ser publicadas de várias formas, as mais comuns são em periódicos ou revistas científicas, que são periodicamente publicadas, podem ser realizadas através de anais de eventos, produzidas através de patentes, também podem ser publicadas como forma de livros ou capítulos de livros.

Diante disto, o presente trabalho visa apresentar de forma quantitativa a produção acadêmica e científica do GPMAC, bem como avaliar o seu aporte em iniciações científicas, TCC's, Dissertações e Teses como forma de evidenciar a contribuição do grupo para a formação de engenheiros mecânicos na graduação e pós-graduação da Faculdade de Engenharia Mecânica da Universidade Federal do Pará.

\section{METODOLOGIA}

Do ponto de vista do procedimento de coleta, a pesquisa se enquadra como um estudo de caso com caráter quantitativo, amparada por uma pesquisa bibliográfica. Estudo de caso consiste no estudo profundo e exaustivo de um assunto, permitindo seu amplo e detalhado conhecimento (GIL, 2008). O estudo de caso visa à investigação de um caso específico, bem delimitado, contextualizado em tempo e lugar para que se possa realizar uma busca circunstanciada de informações (LÜDKE, 2003; YIN, 2015). A pesquisa bibliográfica utiliza como fontes livros, teses, monografias, publicações (jornais, revistas, anais de congressos), meios audiovisuais e material cartográfico (SANTOS, 2000; LAKATOS e MARCONI, 2010).

Como base de dados para a pesquisa foi consultado na Plataforma Lattes o currículo do professor responsável pelo Grupo atualmente (Plataforma Lattes, 2021). Com base nesta análise se iniciou o processo de observação das atividades desenvolvidas pelo grupo, em especial o que se refere à Projetos de Iniciação Científica, Trabalhos de Conclusão de Curso, Dissertações e Teses, bem como as produções acadêmicas e patentes geradas através destes estudos. Trabalhos fora do eixo temático do grupo não foram contabilizados.

Foi feita uma quantificação dos alunos que participaram do grupo com Iniciação Científica, TCC's, Dissertações e Teses e foi realizada uma correlação entre os alunos que participaram de mais de uma dessas atividades em laboratório.

Para melhor ilustrar os resultados se fez o uso de gráficos e tabelas. Os gráficos foram feitos utilizando-se o OriginLab 9 e o Excel pertencente ao pacote Microsoft Office 2019 foi usado para se trabalhar os dados obtidos.

\section{RESULTADOS E DISCUSSÃO}

\subsection{Participação do GPMAC na formação de discentes}

Ao todo 103 discentes passaram pelo grupo desenvolvendo atividades referentes à compósitos sustentáveis, distribuídos entre Iniciação Científica, TCC's, Dissertações de Mestrado ou Teses de Doutorado. Dentre estas atividades a Iniciação Científica é uma das mais importantes para despertar o interesse pelo desenvolvimento de pesquisas e atividades laboratoriais dos alunos de graduação, com isto os dados coletados também mostram que uma parte destes alunos seguiram no GPMAC para realizar TCC's, Dissertações de mestrado e Doutorado. A Tabela 1 mostra basicamente esta relação entre 
a quantidade total de alunos de iniciação científica e a quantidade que prosseguiu no grupo para fazer trabalhos de conclusão de curso, bem como os demais alunos que realizaram mais de uma atividade acadêmica com o grupo.

Tabela 1 - Quantidade da produção científica, de alunos que participaram de uma ou mais atividades acadêmicas junto ao GPMAC e os projetos que envolvem o grupo.

\begin{tabular}{l|c}
\hline \multicolumn{1}{c|}{ Atividade em laboratório } & Quantidade de alunos \\
\hline Alunos de IC & 33 \\
\hline Alunos de IC e TCC & 5 \\
\hline Alunos de IC e Mestrado & 4 \\
\hline Alunos de IC e Doutorado & 1 \\
\hline Alunos de TCC & 45 \\
\hline Alunos de TCC e Mestrado & 3 \\
\hline Alunos de Mestrado & 35 \\
\hline Alunos de Mestrado e Doutorado & 1 \\
\hline Alunos de IC, mestrado e Doutorado & 1 \\
\hline Alunos de Doutorado & 2 \\
\hline Artigos completos publicados em periódicos & 50 \\
\hline Capítulos de livros publicados & 8 \\
\hline Trabalhos completos publicados em anais de congressos & 127 \\
\hline Resumos expandidos publicados em anais de congressos & 1 \\
\hline Resumos publicados em anais de congressos & 21 \\
\hline Artigos aceitos para publicação & 2 \\
\hline Patente & 6 \\
\hline Projetos de Pesquisa & 11 \\
\hline Projeto de extensão & 2 \\
\hline
\end{tabular}

Fonte: Autoria própria (2021).

Das produções referentes à trabalhos de conclusão de curso de graduação e pósgraduação (TCC's, Dissertações de mestrado e Tese de doutorado) o quantitativo de produção no grupo é de 45, 35 e 2 respectivamente. Analisando apenas a quantidade de trabalhos de conclusão se pode notar que é mais abrangente a produção de TCC's, com $54,88 \%$, porém a quantidade de alunos que fizeram dissertação de mestrado também é bastante considerável com $42,68 \%$ tendo em vista a que a quantidade de alunos ingressantes pelo processo habitual de seleção no mestrado acadêmico, que varia entre 40 a 50 alunos por ano, é menor do que os alunos ingressantes na graduação que é de 90 alunos ao ano. Para o doutorado o grupo conta com 2 (2,44\%) alunos concluídos. 
Figura 1 - Trabalhos de Conclusão de curso, dissertações e teses desenvolvidas no GPMAC.

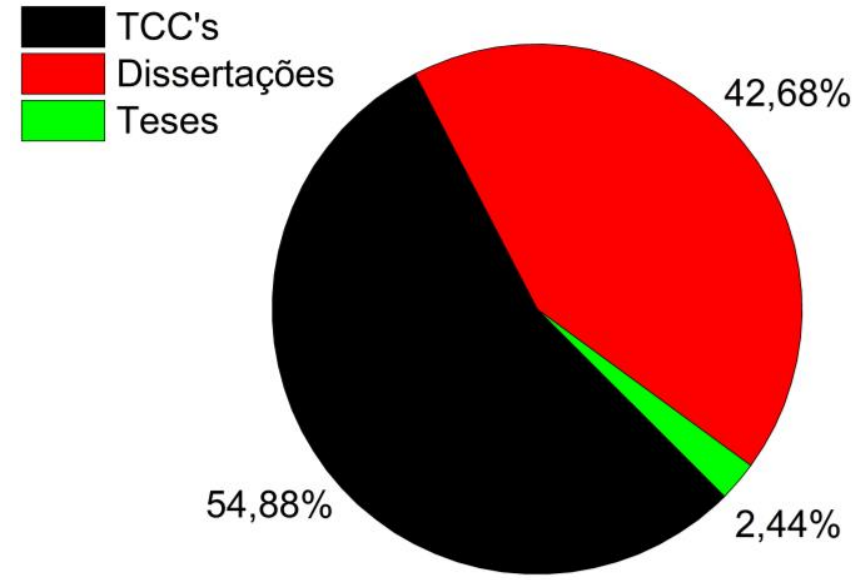

Fonte: Autoria própria (2021).

Visualizando apenas o processo de formação continuada através da pós-graduação, Lordelo \& Argôlo (2015) em sua pesquisa, afirmou que a iniciação científica na graduação influencia positivamente na pós-graduação, pois ela antecipa a continuidade da formação pós-graduada, com grande vantagem para aqueles que tiveram experiências com a pesquisa institucional na graduação em comparação àqueles que não tiveram essa experiência. Neste contexto, a Figura 2 apresenta a relação de alunos que participaram da Iniciação científica no GPMAC e permaneceram no grupo para fazer pós-graduação (mestrado e/ou doutorado) além de correlacionar também a continuidade das atividades da iniciação científica dos participantes do grupo que também realizaram os seus Trabalhos de Conclusão de Curso.

Figura 2 - Correlação entre alunos que participaram de IC e seguiram nas demais atividades acadêmicas com o GPMAC.

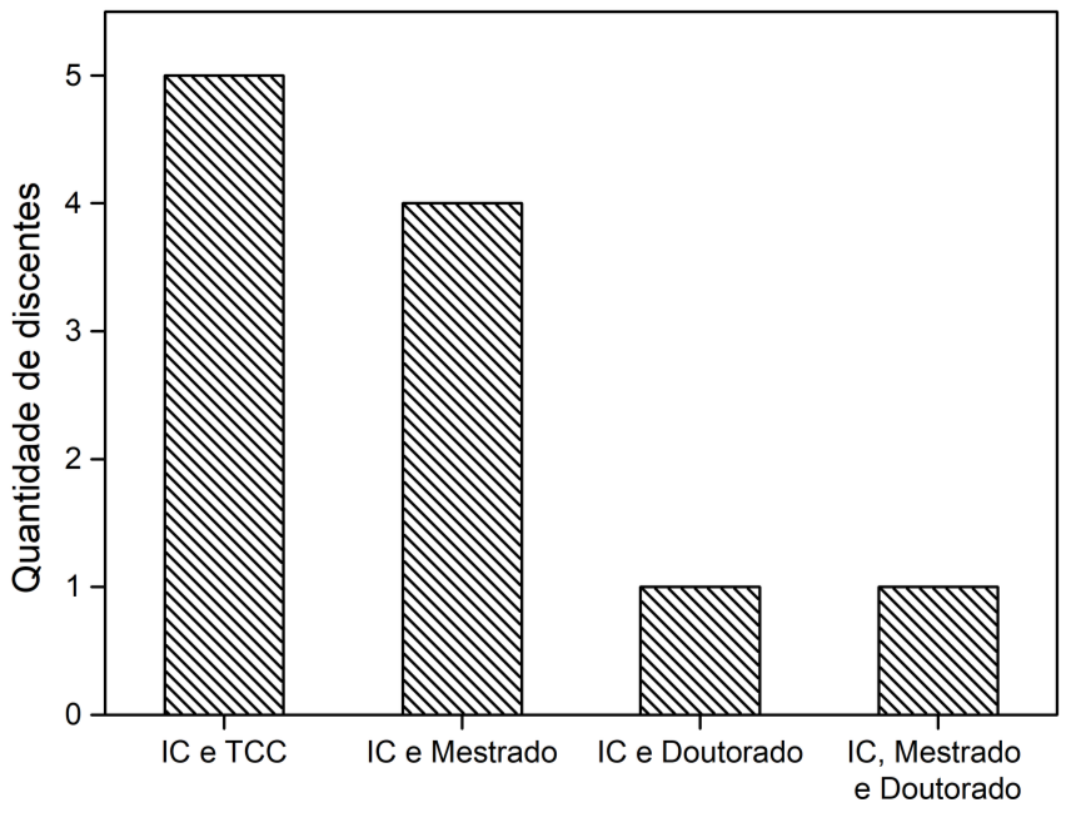

Relação de Iniciação científica com os demais trabalhos

Fonte: Autoria própria (2021). 
Observando a Figura 2, pode-se notar que dentre os 33 alunos de Iniciação Científica que passaram pelo GPMAC, 5 deles, aproximadamente 15,15\%, prosseguiram para fazer o TCC juntamente ao grupo; 4 (12,12\%) também fizeram mestrado, dentre estes 4 , um deles $(3,03 \%)$ percorreu desde a iniciação científica, passando pelo mestrado e doutorado juntamente ao GPMAC.

Analisando somente a quantidade de produção científica publicada do GPMAC, pode-se observar que o Grupo tem foco em publicar por diversos segmentos diferentes, dentre eles se destacam os artigos publicados em periódicos, capítulos de livros, trabalhos completos publicados em anais de eventos e em especial as patentes geradas. A Figura 3 quantifica cada um destes itens acima citados e mostra o percentual de produção para cada um deles.

Figura 2 - Quantidade de publicações científicas por classificação do GPMAC da Faculdade de Engenharia Mecânica da UFPA.

$127(66,49 \%)$

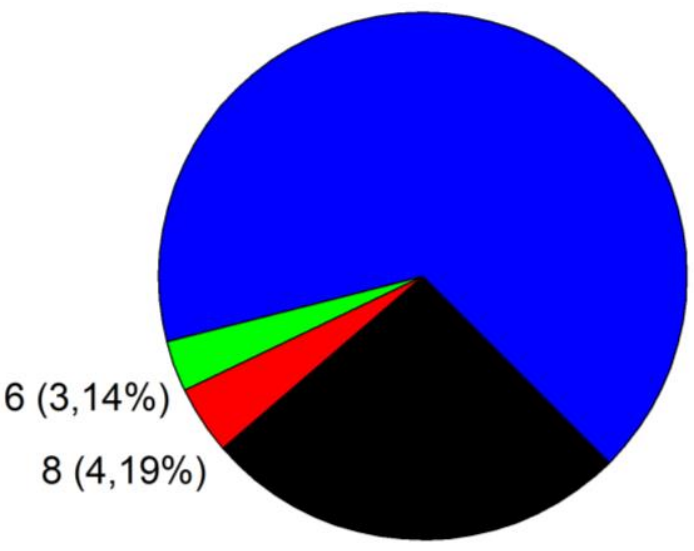

$50(26,18 \%)$

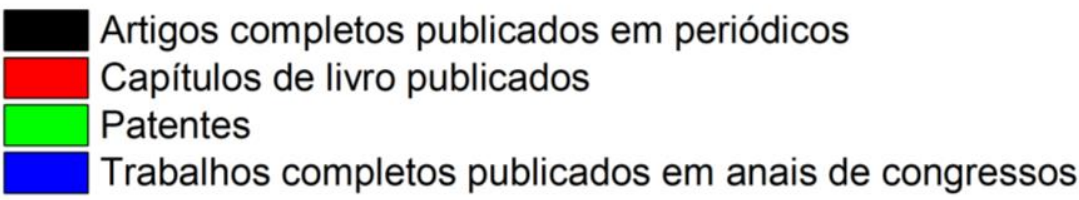

Fonte: Autoria própria (2021).

Na Figura 3 pode-se observar melhor que 66,49\% das publicações do grupo foram feitas em anais de congressos, isso se deve, em especial, pela importante troca de experiencias e interatividade com demais pesquisadores de diversos outros ramos de pesquisa, assim como com grupos que desenvolvem pesquisas similares, isto explana os conhecimentos, em especial, de alunos que ainda não tiveram contato com outros grupos de pesquisa ou que estão iniciando a sua carreira acadêmica.

Artigos científicos publicados com as temáticas utilizadas pelo GPMAC também são importantes, com $26,18 \%$ do total de publicações, esta modalidade apesar de não haver a mesma troca de experiência pessoal exige também de profunda pesquisa, com caráter bastante técnico e direcionamento para atender bem aos critérios estabelecidos por cada revista científica, além de adentrar dentro do eixo temático. Obter resultados satisfatórios, ter boa escrita e correlacionar estes resultados são imprescindíveis em artigos periódicos. 
Pode-se observar também na Figura 3 que 4,19\% das publicações acadêmicas foram de capítulos de livros.

As patentes são métodos de proteção legal temporária sobre a propriedade intelectual disponibilizados no Banco de Patentes do Instituto Nacional de Propriedade Industrial - INPI (FERREIRA, 2009) e servem como uma maneira internacionalmente reconhecida de medir a intensidade do processo inovativo de um país (CRUZ, 2002). Desta forma, a Coordenação de Aperfeiçoamento de Pessoal de Ensino Superior (CAPES) vem estimulando o registro de patentes no Brasil por ser uma das prioridades da Política Industrial e de Comércio Exterior - PITCE (CAPES, 2014). Por conta disto, auxiliado pelo processo criativo do grupo, o GPMAC vem fomentando este tipo de registro conforme pode ser visualizado na Figura 3 e hoje conta com 6 registros de patentes no INPI, o que acrescenta $3,14 \%$ à sua publicação científica.

\section{CONSIDERAÇÕES FINAIS}

Em relação aos produtos de ensino, pesquisa e extensão do Grupo de Pesquisa em Materiais Compósitos - GPMAC da Faculdade de Engenharia Mecânica da UFPA lista-se os seguintes comentários finais:

As atividades de ensino, pesquisa e extensão estão bem evidentes como desenvolvidas pelo grupo de pesquisa.

A Iniciação Científica é uma atividade precursora para as atividades de Trabalho Final de Curso, tanto para a graduação como para a pós-graduação.

Os Trabalhos Finais de Curso de graduação foram em maior quantidade seguida de dissertações de mestrado.

No que se refere a produção bibliográfica, foi observado que a maior quantidade de artigos publicados foi em congressos nacionais e internacionais, seguido de publicações em periódicos.

\subsection{Autorizações/Reconhecimento}

Os autores são responsáveis por garantir o direito de publicar todo o conteúdo de seu trabalho. Se material com direitos autorais foi usado na preparação deste, pode ser necessário obter a devida autorização do detentor dos direitos para a publicação do material em questão.

\section{Agradecimentos}

O presente trabalho foi realizado com apoio da Coordenação de Aperfeiçoamento de Pessoal de Nível Superior-Brasil (CAPES)-Código de Financiamento 001. Os autores agradecem também ao CNPq e Pró-reitoria de Ensino de Graduação da Universidade Federal do Pará, Instituto de Tecnologia e à Faculdade de Engenharia Mecânica.

\section{REFERÊNCIAS}

BRASIL. Ministério da Educação. Plano Nacional de Graduação - PNG. Brasília, 2001 Disponível em: http://portal.mec.gov.br/sesu/arquivos/pdf/png.pdf. Acesso em 21 de abr. 2021.

BROFMAN, P. R. A importância das publicações científicas. Revista Telfract v. 1 n. 1 , 2018. 
CAPES, 2014. Capes estimula aumento de patentes no país. 2014. Disponível em: https://www.uab.capes.gov.br/36-noticias/1141-blank-27049788. Acesso em 25 abr. 2021.

CNPq. RN-005/1993. Programa Institucional de Bolsas de Iniciação Científica PIBIC. Disponível em: http://memoria.cnpq.br/bolsas auxilios/normas/rev/rn0593.pdf. Acesso em: 25 abr. 2021.

CRUZ, C. H. B. A Universidade, a Empresa e a Pesquisa que o País precisa. In: SANTOS, L. W. et al. Ciência, tecnologia e sociedade: o desafio da interação. Londrina: IAPAR, 2002.

DROESCHER, Fernanda Dias; SILVA, Edna Lucia. O pesquisador e a produção científica. Perspectivas em Ciência da Informação, v.19, n.1, p.10-189, 2014.

FACULDADE DE ENGENHARIA MECÂNICA. 2021. Disponível em: http://www.fem.ufpa.br/novofem/. Acesso em: 23 de abr. 2021.

FERREIRA, A. A.; GUIMARÃES, E. R.; CONTADOR, J. C. Patente como instrumento competitivo e como fonte de informação tecnológica. Gest. Prod., São Carlos, v. 16, n. 2, p. 209-221, 2009.

FREITAS, M. F. Q.; SOUZA, J. Pensar a formação e a pesquisa na pós-graduação stricto sensu. Educar em Revista, Curitiba, Brasil, v. 34, n. 71, p. 9-18, 2018.

GIL, Antonio Carlos. Métodos e técnicas de pesquisa social. 6. ed. São Paulo: Atlas, 2008.

INSTITUTO DE TECNOLOGIA - ITEC. 2021. Disponível em: https://itec.ufpa.br/. Acesso em 20 de abr. 2021.

LAKATOS, E. Maria; MARCONI, Marina de Andrade. Fundamentos de metodologia científica. 7. ed. São Paulo: Atlas, 2010.

LÜDKE, Menga. Pesquisa em educação: abordagens qualitativas. 6. ed. São Paulo: EPU, 2003.

MIRANDA, A. C. C.; CARVALHO, E. M. R.; COSTA, M. I. O impacto dos periódicos na comunicação científica Biblos: Revista do Instituto de Ciências Humanas e da Informação, v. 32, n. 1, p. 01-22, 2018.

OLIVEIRA, Glória Aparecida Pereira de. A concepção de egressos de um curso de Pedagogia acerca da contribuição do trabalho de conclusão de curso. 2003.136 f. Dissertação de Mestrado. Faculdade de Educação, Universidade Estadual de Campinas, Campinas, São Paulo, 2003.

SANTOS, Clóvis Roberto dos. Trabalho de conclusão de curso (TCC): guia de elaboração passo a passo. 1. ed. São Paulo: Cengage Learning, 2010. 
UNIVERSIDADE FEDERAL DO PARÁ. Plano de Desenvolvimento Institucional 20162025. Belém, 2016. Disponível em: https://proplan.ufpa.br/index.php/pdi-da-ufpa. Acesso em 20 de abr. 2021.

YIN, Robert K. Estudo de caso: Planejamento e métodos. 5. ed. Porto Alegre: Bookman, 2015.

\title{
SCIENTIFIC AND TECHNOLOGICAL PRODUCTS OF THE COMPOSITE MATERIALS RESEARCH GROUP OF THE MECHANICAL ENGINEERING FACULTY OF UFPA: SCIENTIFIC INITIATION, THE FINAL PAPERS, ARTICLES AND PATENTS
}

\begin{abstract}
The Federal University of Pará (UFPA) has the mission of training people with a sustainable character and generating scientific and technological production to highlight the importance of this theme is fundamental. The Composite Materials Research Group of the Faculty of Mechanical Engineering at UFPA uses these premises in its work with composites reinforced with plant fibers and industrial waste to train its students in all teaching degrees, trying to establish this link from the Scientific Initiation up to the doctorate and this work aims to identify, through a quantitative case study, the contribution of research group scientific and technological production regarding Scientific Initiation, Course Conclusion Papers, Dissertations and Theses, as well as the amount of publication group's scientific research in journals, annals of events, book chapters and in particular regarding patents. It was observed that Scientific Initiation is a precursor activity for the Final Course Work activities, both for undergraduate and graduate students. The Final Papers of the Undergraduate Course were in greater quantity followed by master's dissertations. With regard to bibliographic production, it was observed that the largest number of articles published was in national and international congresses, followed by publications in journals. And the technological innovation products of through patents was $3.14 \%$ of the total of publications.
\end{abstract}

Keywords: Sustainability, Scientific and technological products, Research group. 